Original Article

\title{
Efficacy and Safety of Bone Wax to Reduce Sternal Bleeding Following Coronary Bypass Surgery
}

\author{
Ahmet C Özdemir, MD, and Koray Aykut, MD
}

\begin{abstract}
Objective: To elucidate the effect of bone wax on postoperative bleeding, infection and wound healing.

Methods: This study included two independent groups, consisting of 94 patients who were treated with bone wax and 90 patients who received nothing after median sternotomy and just before sternal closure. Demographic and postoperative data were recorded. Both groups were compared with respect to the amount of postoperative drainage, blood transfusion requirement, re-exploration because of bleeding, and mortality rates.

Results: Demographic measurements did not differ between the groups. In the first two months of the postoperative period, mediastinitis was not seen in any of the patients in both groups. Superficial wound infection was detected in six patients $(6.4 \%)$ in Group A. Eight patients (8.9\%) suffered from superficial wound infection in Group B (p >0.05). In the first postoperative 24 hours, the average amount of postoperative drainage was $536.89 \mathrm{ml}$ in Group A, whereas it was $529.67 \mathrm{ml}$ in Group B $(p>0.05)$. Three patients in both groups died in the early phase of the postoperative period $(p>0.05)$.

There was not any statistically significant difference between groups considering bleeding quantity, mortality, re-exploration, amount of blood used and deep sternal infection.

Conclusion: Bone wax does not reduce bleeding on sternal sides. No evidence was found that application of bone wax causes deep sternal infection in patients having median sternotomy for coronary bypass surgery.
\end{abstract}

Keywords: sternotomy, mediastinitis, bone wax, deep sternal wound infection

\section{Introduction}

Median sternotomy is a quickly and easily applied incision method that is commonly used during openheart surgery, which allows better operating field exposure and easy access into cardiac compartments

Department of Cardiovascular Surgery, Private Ege Hospital, Denizli, Turkey

Received: October 16, 2012; Accepted: January 29, 2013

Corresponding author: Ahmet Coşkun Özdemir, MD. Servergazi Mah. 211. Sok. Platin Konutlar1 C Blok Daire 5, 20055 Denizli, Turkey

Email: drcozdemir@yahoo.com

(C)2014 The Editorial Committee of Annals of Thoracic and Cardiovascular Surgery. All rights reserved. facilitating cardiac cannulation. Sternal bleeding sometimes necessitates the use of large amounts of blood and blood products and rarely leads to re-exploration. Since the introduction of median sternotomy which allows access into intrathoracic organs, sternal wound infection and dehiscence have been reported to occur in only $0.5 \%-5 \%$ of the operated cases. Various studies have shown rates of mediastinitis, and mortality as $1 \%-2.5 \%$, and $15 \%-50 \%$, respectively. ${ }^{1)}$ The most common method to reduce or stop the sternal bleeding is to apply bone wax on sternal sides.

Bone wax is a mixture of beeswax, paraffin wax and isopropyl palmitate which is used to control injured bone surface bleeding. Bone wax stops bleeding by blocking up the holes and forms a mechanic barrier in spongy 
tissues on sternal sides. It reduces bleeding so that the left internal mammary artery (LIMA) can be harvested easily with decreased use of blood and blood products. However, it is also pointed out that bone wax has an alleviating effect on infections and wound healing inhibitors. ${ }^{2)}$ In the present study, we aimed to investigate abovementioned effects of bone wax.

\section{Materials and Methods}

This study was approved by the local Institutional Review Board. Written informed consent was obtained from all subjects, a legal surrogate, the parents or legal guardians.

One hundred eighty-four patients who had undergone coronary artery bypass surgery with open-heart technique between January 2009 and April 2009 were prospectively enrolled in the study (Table 1). First-time candidates for sternotomy with normal preoperative blood coagulation test (aPTT, PT, INR) results who were not taking any antiaggregant or anticoagulant medicine during the last week were included in the study. A total of 13 patients were excluded from the study. Seven of them were off-pump coronary bypass cases. One patient with renal failure and five patients with continuing antiagregant-anticoagulant therapy in the last week were also excluded. The patients were randomly divided into two groups. The randomization was performed by a study coordinator, who was blinded to the allocation of patients and used a computer-generated random number sequence. For patients in Group A $(\mathrm{n}=94)$, bone wax was applied on cut surfaces just after the sternotomy and immediately before sternal closure. For patients in Group B $(n=90)$, any hemostatic material or method was not used.

\section{Surgical technique}

Preoperatively, as a standard procedure for all patients operative field was disinfected with polyvinylpyrrolidone iodine application and covered with a sterile drape without leaving the chest area open. After median sternotomy, in order to prevent bleeding the top and bottom parts of sternal sides were electrocauterized, and bone wax (Cliniwax 2.5g, YÜCEL Medikal, Istanbul, Turkey) was applied only in Group A. Cardiopulmonary bypass was instituted with aortocaval cannulation in all patients. Just before the sternal closure, wax was applied on cut edges once again for group A, and sternal closure was done with stainless steel wires (No.5). Following anesthesia, cefazolin ( $1 \mathrm{~g}$ tid IV) therapy was initiated for 48 hours.
Table 1 Demographic profile and the risk factors of the patients

\begin{tabular}{lcc}
\hline & Group A & Group B \\
\hline No. of patients & 94 & 90 \\
Mean age (years) & $58.2 \pm 8.4$ & $61.6 \pm 6.4$ \\
Female & $51(54.2 \%)$ & $54(60 \%)$ \\
Smoking & $45(47.8 \%)$ & $46(51.1 \%)$ \\
COPD & $8(8.5 \%)$ & $10(11.1 \%)$ \\
Diabetes Mellitus & $42(44.6 \%)$ & $48(53.3 \%)$ \\
Hipertension & $50(53.1 \%)$ & $6(51.1 \%)$ \\
LIMA harvest & $94(100 \%)$ & $90(100 \%)$ \\
Mean no. of bypass grafts & $3.08 \pm 0.3$ & $3.15 \pm 0.6$ \\
Mean CPB time & $82.7 \pm 6.0$ & $84.2 \pm 4.9$ \\
Left ventricle EF(\%) & $48.4 \pm 2.6$ & $51.5 \pm 4.8$ \\
\hline COPD: chronic obstructive pulmonary disease; LIMA: left \\
internal mammarian artery ; CPB: cardiopulmonary bypass; \\
EF: elongation factor
\end{tabular}

Both groups were compared with respect to the amount of postoperative drainage, blood transfusion requirement, re-exploration because of bleeding, and mortality rates. Superficial wound infection was defined as sternotomy incision discharge limited to the skin or the subcutaneous tissue with no sternal instability or dehiscences. Mediastinitis or deep sternal infection was defined as sternal instability or dehiscences with purulent discharge or positive microbiology.

Each of the following was an indication for reexploration: (i) drainage of $>500 \mathrm{~mL}$ during the first hour, $>400 \mathrm{~mL} /$ hour during first two hours, $>300 \mathrm{~mL} /$ hour during first three hours, or more than $1000 \mathrm{~mL}$ in total during the first four hours; (ii) subtle, continuous bleeding throughout the first 12 hours, leading to a total bleeding exceeding $100 \mathrm{~mL} /$ hour; (iii) sudden massive bleeding; and (iv) obvious signs of cardiac tamponade.

\section{Statistical analysis}

Data were analyzed using the Statistical Package for Social Sciences (SPSS) software (Version 19.0 for Windows; SPSS Inc., Chicago, Illinois, USA). Results were analyzed with independent two samples T-test for continuous variables and chi-square test, when appropriate, for discrete variables. All differences associated with a chance probability of 0.05 or less were considered statistically significant. Continuous variables are presented as mean $\pm \mathrm{SD}$.

\section{Results}

In the first two months of the postoperative period, 
Table 2 Comparison of complications between the patients in Group A and Group B

\begin{tabular}{lccc}
\hline & Group A & Group B & p value \\
\hline No. of patients & 94 & 90 & \\
Postoperative drainage (ml) & $536.89 \pm 218.11$ & $529.67 \pm 214.42$ & .822 \\
Blood Transfusion (unit) & $2.15 \pm 0.81$ & $2.29 \pm 0.97$ & .293 \\
Superficial wound infection & $6(6.4 \%)$ & $8(8.9 \%)$ & .522 \\
Mediastinitis & $0 \%$ & $0 \%$ & \\
Reexploration for bleeding & $1(1.1 \%)$ & $2(2.2 \%)$ & .615 \\
Sternal dehiscence & $3(3.2 \%)$ & $2(2.2 \%)$ & .686 \\
Mortality & $3(3.2 \%)$ & $3(3.3 \%)$ & .957 \\
\hline
\end{tabular}

mediastinitis was not seen in any of the patients in both groups. A superficial wound infection was detected in six patients $(6.4 \%)$ in Group A. Eight patients (8.9\%) suffered from superficial wound infection in Group B $(p>0.05)$. In the first postoperative 24 hours, the average amount of postoperative drainage was $536.89 \mathrm{ml}$ in Group A, whereas it was $529.67 \mathrm{ml}$ in Group B ( $\mathrm{p}>0.05)$. Three patients in both groups died because of acute renal failure and low cardiac output in the early phase of the postoperative period $(\mathrm{p}>0.05)$ (Table 2). Wound infections were treated with empirical antibiotic therapy (ampicillin/sulbactam and ciprofloxacin). One patient $(1.1 \%)$ in Group B underwent sternum revision due to sternal dehiscence. The other four patients with sternal dehiscence recovered well with the help of chest binder support.

\section{Discussion}

The number of cardiac operations increases in all countries and parallel to this, the problems of sternotomy healing leads cardiac surgeons to search for new options and use several new techniques and materials. In advanced age group, especially in female patients sternal bone tissues and mineral loss increase the risk of deep sternal infection and osteomyelitis. ${ }^{1)}$ Mediastinitis seen after coronary bypass surgery is a frequent complication with high mortality rates. In a study performed with 7602 patients who had undergone coronary bypass surgery, deep sternal wound infection, and mortality rates were found to be $0.59 \%$, and $9.1 \%$, respectively. ${ }^{2)}$ In various studies, mortality rates associated with mediastinitis have ranged between $10 \%$ and $47 \% .{ }^{3)}$ Advanced age, male gender, NYHA (New York Heart Association) class $\geq 3$, left main coronary stenosis, bilateral internal mammary artery harvesting, body mass index over $30 \mathrm{~kg} / \mathrm{m}^{2}$, chronic obstructive pulmonary disease, smok- ing, diabetes mellitus, prolonged mechanical ventilation, re-exploration due to bleeding and massive blood transfusion are found to be the independent risk factors for mediastinitis. ${ }^{4-6)}$

Bone wax is a mixture of beeswax, paraffin wax and isopropyl palmitate which is used to control injured bone surface bleeding. It is used to reduce the bleeding on sternal sides in cardiac surgery providing bloodless surgical exposure with resultant lesser requirement for blood transfusion. LIMA harvesting can be done faster and more comfortable with the reduction of bleeding on sternal sides. Bone wax stops bleeding by blocking up the holes, and forms a mechanic barrier in spongy tissues on sternal sides. However, some studies have demonstrated harmful effects of bone wax application, in addition to its above-mentioned benefits. In an autopsy case series of 18 patients, even after 10 years, it was verified that bone wax was not absorbed, and 17 patients had chronic inflammation. ${ }^{7)}$ Histologic examinations in animal models have demonstrated that application of bone wax prevents bone healing and creates fibrotic scar tissue. ${ }^{8)}$ Various studies in the literature have demonstrated that application of bone wax increased the risk of mediastinitis in patients following sternotomy. However, Bhatti, et al. reviewed 276 studies in the literature concerning the association between bone wax and mediastinitis, but they could not find any literature study suggesting that bone wax application in humans directly increases the risk of mediastinitis.9) In a study by Milano, et al. performed with 6459 patients in which they investigated the risk factors of mediastinitis, the authors could not demonstrate that bone wax application increases the risk of mediastinitis. ${ }^{10)}$ Steingrimsson, et al. investigated the development of sternocutaneous fistulas in cardiac surgery patients. In this study, they found that the presence of sternal wound infection, kidney failure, smoking and bone wax application were independent risk factors in 
the development of sternocutaneous fistulas. ${ }^{11)}$ Many different materials, such as calcium phosphate cement, absorbable microfibrillation collagen, fibrins and dissoluble alkylene oxide copolymers were used instead of bone wax to control the bleeding on sternal sides, and their properties were compared with bone wax. ${ }^{12-14)}$

In our study, any statistically significant difference was not found between groups considering the quantity of bleeding, amount of blood and blood products used and deep sternal infection. It could not be shown that the application of bone wax significantly reduces bleeding following sternotomy. On the other hand, concerning deep sternal wound infection, a statistically significant difference was not found between two groups. This result does not support the opinion that application of bone wax after median sternotomy increases the risk of deep sternal infection. A very low incidence of adverse events makes it difficult to detect a difference between the two groups. Further studies in larger patient populations are required to assess the side effects of bone wax.

\section{Conclusion}

Bone wax does not reduce bleeding on sternal sides, and as a result, requirement for blood and blood products in patients having median sternotomy for coronary bypass surgery does not decrease. In addition, no evidence was found that application of bone wax causes deep sternal infection in patients having median sternotomy for coronary bypass surgery.

Thus, it is not necessary to apply bone wax to reduce bleeding following median sternotomy in coronary bypass patients. In addition, the application of other materials should be decided after analyzing the actual cost and the long- term effects of these materials.

\section{Disclosure Statement}

None declared. This research received no specific grant from any funding agency in the public, commercial, or not-for-profit sectors.

\section{References}

1) Loop FD, Lytle BW, Cosgrove DM, et al. J. Maxwell Chamberlain memorial paper. Sternal wound complications after isolated coronary artery bypass grafting: early and late mortality, morbidity, and cost of care. Ann Thorac Surg 1990; 49: 179-86; discussion 186-7.

2) Ariyaratnam P, Bland M, Loubani M. Risk factors and mortality associated with deep sternal wound infections following coronary bypass surgery with or without concomitant procedures in a UK population: a basis for a new risk model? Interact Cardiovasc Thorac Surg 2010; 11: 543-6.

3) Salazard B, Niddam J, Ghez O, et al. Vacuum-assisted closure in the treatment of poststernotomy mediastinitis in the paediatric patient. J Plast Reconstr Aesthet Surg 2008; 61: 302-5.

4) Risnes I, Abdelnoor M, Almdahl SM, et al. Mediastinitis after coronary artery bypass grafting risk factors and long-term survival. Ann Thorac Surg 2010; 89: 1502-9.

5) Sachithanandan A, Nanjaiah $P$, Nightingale $P$, et al. Deep sternal wound infection requiring revision surgery: impact on mid-term survival following cardiac surgery. Eur J Cardiothorac Surg 2008; 33: 673-8.

6) Lu JC, Grayson AD, Jha P, et al. Risk factors for sternal wound infection and mid-term survival following coronary artery bypass surgery. Eur J Cardiothorac Surg 2003; 23: 943-9.

7) Sudmann B, Bang G, Sudmann E. Histologically verified bone wax (beeswax) granuloma after median sternotomy in 17 of 18 autopsy cases. Pathology 2006; 38: 138-41.

8) Wellisz T, Armstrong JK, Cambridge J, et al. The effects of a soluble polymer and bone wax on sternal healing in an animal model. Ann Thorac Surg 2008; 85: 1776-80.

9) Bhatti F, Dunning J. Does liberal use of bone wax increase the risk of mediastinitis? Interact Cardiovasc Thorac Surg 2003; 2: 410-2.

10) Milano CA, Kesler K, Archibald N, et al. Mediastinitis after coronary artery bypass graft surgery. Risk factors and long-term survival. Circulation 1995; 92: 2245-51.

11) Steingrímsson $S$, Gustafsson R, Gudbjartsson $T$, et al. Sternocutaneous fistulas after cardiac surgery: incidence and late outcome during a ten-year follow-up. Ann Thorac Surg 2009; 88: 1910-5.

12) Wellisz T, Armstrong JK, Cambridge J, et al. Ostene, a new water-soluble bone hemostasis agent. J Craniofac Surg 2006; 17: 420-5.

13) Kjaergard HK, Trumbull HR. Bleeding from the sternal marrow can be stopped using vivostat patient-derived fibrin sealant. Ann Thorac Surg 2000; 69: 1173-5.

14) Blanche $C$, Chaux A. The use of absorbable microfibrillation collagen to control sternal bone marrow bleeding. Int Surg 1988; 73: 42-3. 